\title{
Electrospun Silk Fibroin Scaffolds with Macropores for Bone Regeneration: An In Vitro and In Vivo Study
}

\author{
Sook Young Park, M.S., ${ }^{1, *}$ Chang Seok Ki, Ph.D., 2,3, ${ }^{\star}$ Young Hwan Park, Ph.D., ,3, ${ }^{*}$ \\ Hong Moon Jung, M.S., ${ }^{4}$ Kyung Mi Woo, M.D., Ph.D., ${ }^{4}$ and Hyun Jeong Kim, M.D., Ph.D., ${ }^{\star}$
}

We developed three-dimensional electrospun silk fibroin (ESF) scaffolds with controllable pore size. The purpose of this study was to evaluate ESF scaffolds with pores (P-ESF) for bone regeneration via in vitro and in vivo studies, with a comparison to a commercially available porous three-dimensional polylactic acid (PLA) scaffold. P-ESF supported significantly higher proliferation and alkaline phosphatase activity of osteoblasts than PLA in vitro $(p<0.05)$. Moreover, higher expression levels of activated adhesion-related proteins, including focal adhesion kinase, were observed in the P-ESF than in PLA, as confirmed by western blot analyses. Microcomputed tomography revealed that $78.30 \%$ of the original bone volume was attained in the P-ESF implantation group at 7 weeks after critical bone defect formation in rat calvaria. Comparatively, the PLA implantation group showed only $49.31 \%$. Histological evaluation also showed new bone tissue formation upon P-ESF implantation. Taken together, the P-ESF scaffold may be a good bone substitute for bone regeneration.

\section{Introduction}

B ONE REMODELING is a dynamic process not only in pathological but also under normal conditions in the body. Bone has a three-dimensional (3D), mineralized structure. Bone is continuously degraded and regenerated by interaction between osteoclasts and osteoblasts. ${ }^{1-3}$ However, adequate bone regeneration may be limited when the defect is beyond the natural regeneration capacity.

Clinically, implantations of autograft, allograft, xenograft, metallic implants, and artificial synthetic materials are used for large defects. Until now, the best way for repairing a critical bone defect was autograft. However, the autograft has several limitations, including the necessity of another operation besides the defect area and functional impairment of the donor site. Grafting materials from others can also cause risk of serious systemic infection from donors, immune rejection, chronic immune responses, and toxicity. Therefore, a wide variety of novel materials and methods are still being developed and suggested based on tissue engineering techniques for bone regeneration. ${ }^{4-6}$

Silk is an organic fiber produced by silk worms, and is one of the strongest and toughest materials known. Structurally, native silkworm silk protein from Bombyx mori consists of a core structural fibroin protein surrounded by sericin, a gluelike protein. Fibroin is well known for being biocompatible and cytocompatible, and thus it is a promising biomaterial for bone regeneration. ${ }^{7-11}$

Recently, electrospinning using a high electric field has become popular for making biomaterials. This technique can provide many kinds of scaffolds with a large surface area and natural extracellular matrix (ECM)-like structure, which is vital for cellular attachment and proliferation. ${ }^{12,13}$ The electrospun 3D scaffolds (electrospun silk fibroin [ESF]) exhibit a high efficiency for cell attachment in early stages of cell culture, and its highly porous structure is beneficial to nutrient or gas exchange for cells. ${ }^{14-16}$ Usually, electrospun scaffolds have been limited to a two-dimensional (2D) sheet because of the conventional collecting method using a metal plate or mandrel. Recently, we developed a pore size-controllable 3D ESF (P-ESF) via a salt-leaching method where various sized $\mathrm{NaCl}$ particles act to control pores in the middle of a silk fibroin mass. ${ }^{17,18}$

In this study, we compared pore size-matched P-ESF with a commercially available sponge-type 3D polylactic acid (PLA) scaffold using an osteoblast cell line, MC3T3-E1, in vitro in terms of cellular viability, proliferation, and osteoblast differentiation. Moreover, we applied the P-ESF

\footnotetext{
${ }^{1}$ Department of Dental Anesthesiology and Dental Research Institute, School of Dentistry, Seoul National University, Seoul, Republic of Korea.

${ }^{2}$ Department of Biosystems and Biomaterials Science and Engineering, Seoul National University, Seoul, Republic of Korea.

${ }^{3}$ Research Institute for Agriculture and Life Sciences, Seoul National University, Seoul, Republic of Korea.

${ }^{4}$ Department of Cell and Developmental Biology, School of Dentistry, Seoul National University, Seoul, Republic of Korea.

*These authors contributed equally to this work.
} 
scaffold to critical bone defects of rat calvaria. We also compared bone formation between P-ESF and PLA scaffolds using microcomputed tomography $(\mu \mathrm{CT})$ and hematoxylin and eosin (H\&E) staining at 7 weeks after bone defect surgery. Nonporous ESF (N-ESF) scaffold, normal calvaria, and defected calvaria were also evaluated to compare the bone regeneration of P-ESF within in vivo experiments.

\section{Materials and Methods}

\section{Materials}

Bombyx mori cocoons were boiled in aqueous sodium carbonate solution $(0.2 \%, \mathrm{w} / \mathrm{v})$ with sodium oleate $(0.3 \%, \mathrm{w} / \mathrm{v})$ for $1 \mathrm{~h}$ and washed with distilled water to remove sericin. Then the degummed cocoons were dissolved in a ternary solvent of $\mathrm{CaCl}_{2} / \mathrm{H}_{2} \mathrm{O} / \mathrm{EtOH}$ (mole ratio: $1 / 8 / 2$ ) at $85^{\circ} \mathrm{C}$ for $3 \mathrm{~min}$, and the SF aqueous solution was dialyzed against distilled water for 3 days and lyophilized. The other solvents and chemicals were used without further purification.

\section{Scaffold preparations}

Electrospinning and fabrication. At first, SF fiber dispersion was obtained by electrospinning. The $13 \%(\mathrm{w} / \mathrm{v})$ dope solution was prepared by dissolving the lyophilized SF in formic acid. The resulting solution was poured into a syringe with a 22-G needle connected to a DC high voltage power supply (Chungpa EMT, Seoul, Korea). The entire electrospinning apparatus and details were designed from previous reports. ${ }^{17,18} \mathrm{~A}$ methanol coagulation bath, which collected and recrystallized the electrospun SF fibers, was located $20 \mathrm{~cm}$ below the needle. The dope solution feed rate was accurately controlled to match the spin speed and the applied voltage was $13 \mathrm{kV}$.

The collected SF fiber dispersion was moved into a glass vessel. Then methanol (dispersion medium) was exchanged with 1,4-dioxane and sodium chloride particles (porogen) with varying diameters, ranging from 50 to 100,100 to 200 , and 300 to $500 \mu \mathrm{m}$. The amount of added salt was $0.15 \mathrm{~g} / \mathrm{mL}$ irrespective of particle size. Subsequently, the mixture was gently stirred to mix the particles with the SF fibers and then molded in a glass tube (I.D.: $6 \mathrm{~mm}$ ). The molded SF fiber assembly was lyophilized and subsequently exposed to glutaraldehyde vapor in a sealed desiccator for 1 day. Then the molded SF fiber assembly was detoxified with $0.1 \mathrm{M}$ glycine buffer ( $\mathrm{pH}$ 9.2) and subsequently washed with phosphate-buffered saline several times. ${ }^{19}$ The final shape of the SFS was a disk form (6 $\mathrm{mm}$ diameter and $1.5 \mathrm{~mm}$ thick). The porous but not fibrous poly(lactic acid) (PLA) scaffolds of same pore dimension (BD 3-D Scaffold OPLA ${ }^{\circledR}$, cat. no. REF 354614; BD Falcon, Sparks, MD) were used as a control group. Each sample designation is listed in Table 1.

Morphological structure analysis. The morphological structure of the scaffold was observed via scanning electron microscopy. The diameter of the SF fiber was determined using image software (Adobe Photoshop, San Jose, CA) and the pore size was determined by calculating the equivalent circular diameter after measuring the pore cross-sectional area. Equivalent circular diameter denotes the diameter of a circle that has the same area as the pore cross-section.
Table 1. Various Pore-Sized Fibrous Silk Fibroin Scaffolds Used in This Study

\begin{tabular}{ll}
\hline Scaffolds & \\
\hline N-ESF & $\begin{array}{c}\text { Silk fibroin scaffold without pores } \\
\text { (NaCl particles were not added) } \\
\text { Silk fibroin scaffold with small pores } \\
\text { (NaCl particle size: } 50-100 \mu \mathrm{m})\end{array}$ \\
S-ESF & $\begin{array}{l}\text { Silk fibroin scaffold with medium pores } \\
\text { (NaCl particle size: } 100-200 \mu \mathrm{m})\end{array}$ \\
M-ESF (P-ESF) & $\begin{array}{c}\text { Silk fibroin scaffold with large pores } \\
\text { (NaCl particle size: } 300-500 \mu \mathrm{m})\end{array}$ \\
L-ESF & $\begin{array}{c}\text { Commercial PLLA scaffold purchased } \\
\text { from BD Falcon (pore size: } 100-200 \mu \mathrm{m})\end{array}$ \\
PLA &
\end{tabular}

N-ESF, nonporous electrospun silk fibroin; S-ESF, small ESF; M-ESF, medium ESF; P-ESF, ESF with pores; L-ESF, large ESF; PLA, polylactic acid.

Porosity and water uptake measurement. The porosity measurement of ESF was calculated by a previously reported method. ${ }^{20}$ The bulk and fiber densities $\left(\rho_{\mathrm{B}}\right.$ and $\left.\rho_{\mathrm{F}}\right)$ were determined by Equations (1) and (2), respectively.

$$
\begin{gathered}
\rho_{\mathrm{B}}=\frac{4 m_{1}}{D^{2} t} \\
\rho_{\mathrm{F}}=\frac{m_{1}}{m_{1}-m_{2}} \rho_{\mathrm{H}}
\end{gathered}
$$

where $m_{1}$ and $m_{2}$ are the masses of ESFs in air and $n$-hexane after a brief vacuum, respectively, $D$ is diameter, and $t$ is thickness. $\rho_{\mathrm{H}}$ is the density of $n$-hexane at $25^{\circ} \mathrm{C}$. From $\rho_{\mathrm{B}}$ and $\rho_{\mathrm{F}}$, the porosity could be calculated as shown in Equation (3).

$$
\text { Porosity }(\%)=\left(1-\frac{\rho_{\mathrm{B}}}{\rho_{\mathrm{F}}}\right) \times 100
$$

On the other hand, water uptake was determined by Equation (4) after soaking the ESF in phosphate-buffered saline for $1 \mathrm{~h}$.

$$
\text { Water uptake }(\%)=\frac{m_{\mathrm{w}}-m_{\mathrm{d}}}{m_{\mathrm{d}}} \times 100
$$

where $m_{\mathrm{d}}$ and $m_{\mathrm{w}}$ are the masses in dry and wet states, respectively.

\section{Cell culture and in vitro test}

MC3T3-E1 clone 4 cells were cultured in $\alpha$-minimum essential medium supplemented with $10 \%(\mathrm{v} / \mathrm{v})$ fetal bovine serum and $1 \%(\mathrm{v} / \mathrm{v})$ antibiotic/antimycotic solution at $37^{\circ} \mathrm{C}$ in a humidified, $5 \% \mathrm{CO}_{2}$ atmosphere. After harvest, cells were seeded directly on each sterilized scaffold $\left(2.5 \times 10^{5}\right.$ cells $\left./ \mathrm{cm}^{2}\right)$. Some of the samples were observed by scanning electron microscope (SEM, JEM-T300; Jeol, Tokyo, Japan) after each culture period.

The remaining samples were tested via DNA content, MTT, and alkaline phosphatase (ALP) assays to measure the cell proliferation and mineralization ( $n=5$ per group). DNA of cells on scaffolds was isolated following the kit protocol (Qiagen, Valencia, CA) and the concentration was measured 
using a spectrometer (ND-1000; NanoDrop, Wilmington, DE). For MTT assay, samples were incubated at $37^{\circ} \mathrm{C}$ in $5 \% \mathrm{CO}_{2}$ for $4 \mathrm{~h}$ in serum-free $\alpha$-minimum essential medium supplemented with $0.5 \mathrm{~g}$ of 3-(4,5-dimethylthiazol-2-yl)2,5-diphenyltetrazolium bromide and the purple formazan was extracted using $0.04 \mathrm{M} \mathrm{HCl}$ in 2-propanol. The extracted solution was measured at $570 \mathrm{~nm}$ using a UV/VIS spectrophotometer. To observe mineralization, ALP of cells was measured. ALP of samples was extracted according to the ALP assay kit (Takala Bio, Shiga, Japan) protocol and measured at $405 \mathrm{~nm}$.

The other samples were prepared for western immunoblotting to compare adhesion signals between scaffolds. Lysate proteins were separated via $8 \%$ sodium dodecyl sulfate-polyacrylamide gel electrophoresis gel and transferred to polyvinylidene fluoride (PVDF) membranes using an I-Blot transfer machine (Invitrogen, Carlsbad, CA) for $6 \mathrm{~min}$. The membranes were reacted with primary antibodies for $12 \mathrm{~h}$ at $4{ }^{\circ} \mathrm{C}$. The antibodies used were anti-integrin $\alpha 5(\mathrm{H}-104)$, -c-Src (B-12), -phospho-Tyr925 focal adhesion kinase (FAK) (Santa Cruz Biotech, Santa Cruz, CA), -phospho-Tyr118 paxillin, -phospho-Tyr416 c-Src, -phospho-ERK1/2, -ERK1/2 (Cell Signaling Technology, Beverly, MA), -FAK, -paxillin (BD Bioscience, San Jose, CA), and -phospho-Tyr397 FAK (Chemicon, Temecula, CA). After reaction with the primary antibody, the membrane was incubated again with secondary antibody (mouse or rabbit) for $1 \mathrm{~h}$ at room temperature. The HRP activity was measured using an ECL kit (Pierce, Rockford, IL). Detailed information of antibodies for western blotting is given in Supplemental Material (available online at www.liebertonline.com).

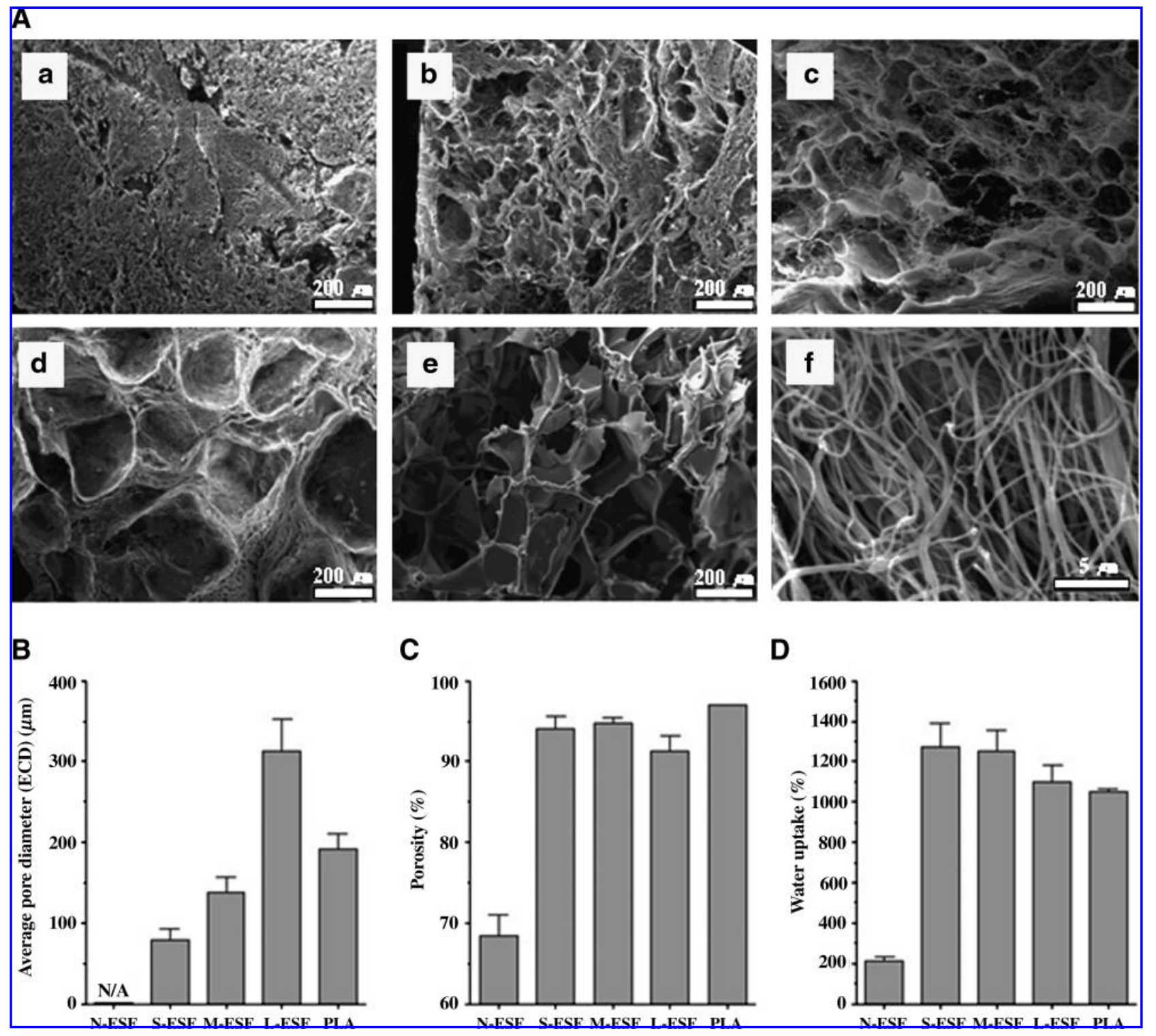

FIG. 1. Characteristics of various pore-sized fibrous silk fibroin scaffolds and sponge-type PLA scaffold. (A) Scanning electron microscope photographs of scaffold cross-sections: (A-a) N-ESF, (A-b) S-ESF, (A-c) P-ESF (M-ESF), (A-d) L-ESF, and (A-e) PLA purchased from BD Falcon. Also, magnified individual fiber of ESF is shown (A-f). (B) Average pore diameter. (C) Porosity. (D) Water uptake. Data are shown as mean \pm standard error of the mean $(n=4)$. N/A, not available; N-ESF, nonporous electrospun silk fibroin; S-ESF, small ESF; M-ESF, medium ESF; P-ESF, ESF with pores; L-ESF, large ESF; PLA, polylactic acid. 


\section{Animal surgery for in vivo test}

To evaluate the performance in vivo, 24 male SpragueDawley rats (300-350 g) were anesthetized via isofluraneoxygen inhalation (FORENE ${ }^{\mathrm{TM}}$; Abbott AG, Neuhofstrasse, Switzerland). After incision of skin and muscle, the exposed calvaria were punched using a 7.00-mm-diameter drill, and each scaffold was subsequently transplanted. Then the rats were allowed to recover for 7 weeks and sacrificed with $\mathrm{CO}_{2}$ gas to retrieve the calvaria. The entire procedure followed the policy of the Seoul National University Animal Care and Ethics Committee.

\section{Radiographic and histological evaluation}

The rat calvaria specimens were fixed with $10 \%$ formalin for $24 \mathrm{~h}$. Then radiographs of the specimens were taken using $\mu C T$ (SKYSCAN 1072-32, Kontich, Belgium). A total of 359 slices were imaged ( $19.53125 \mu \mathrm{m}$ per slice for 3.4-s exposure) for every specimen, and the pictures were analyzed and used to generate a 3D image of $1024 \times 1024$ pixels using CT reconstruction software from SKYSCAN. The regenerated bone area was quantified using an image analysis program (Image Pro 6.2; Media Cybernetics, Bethesda, MD).

After radiographic testing, samples were decalcified in a $10 \%$ ethylenediaminetetraacetic acid solution, which was changed every day for 3 weeks. Then samples were embedded with paraffin and cut into $6-\mu \mathrm{m}$ sections. The tissue slices were subjected to deparaffinization and hydration steps and then stained with hematoxylin for $10 \mathrm{~min}$ and eosin for $2 \mathrm{~min}$. Histology photographs were taken under a BX-51 microscope (OLYMPUS, Tokyo, Japan) and analyzed (Image Pro 6.2).

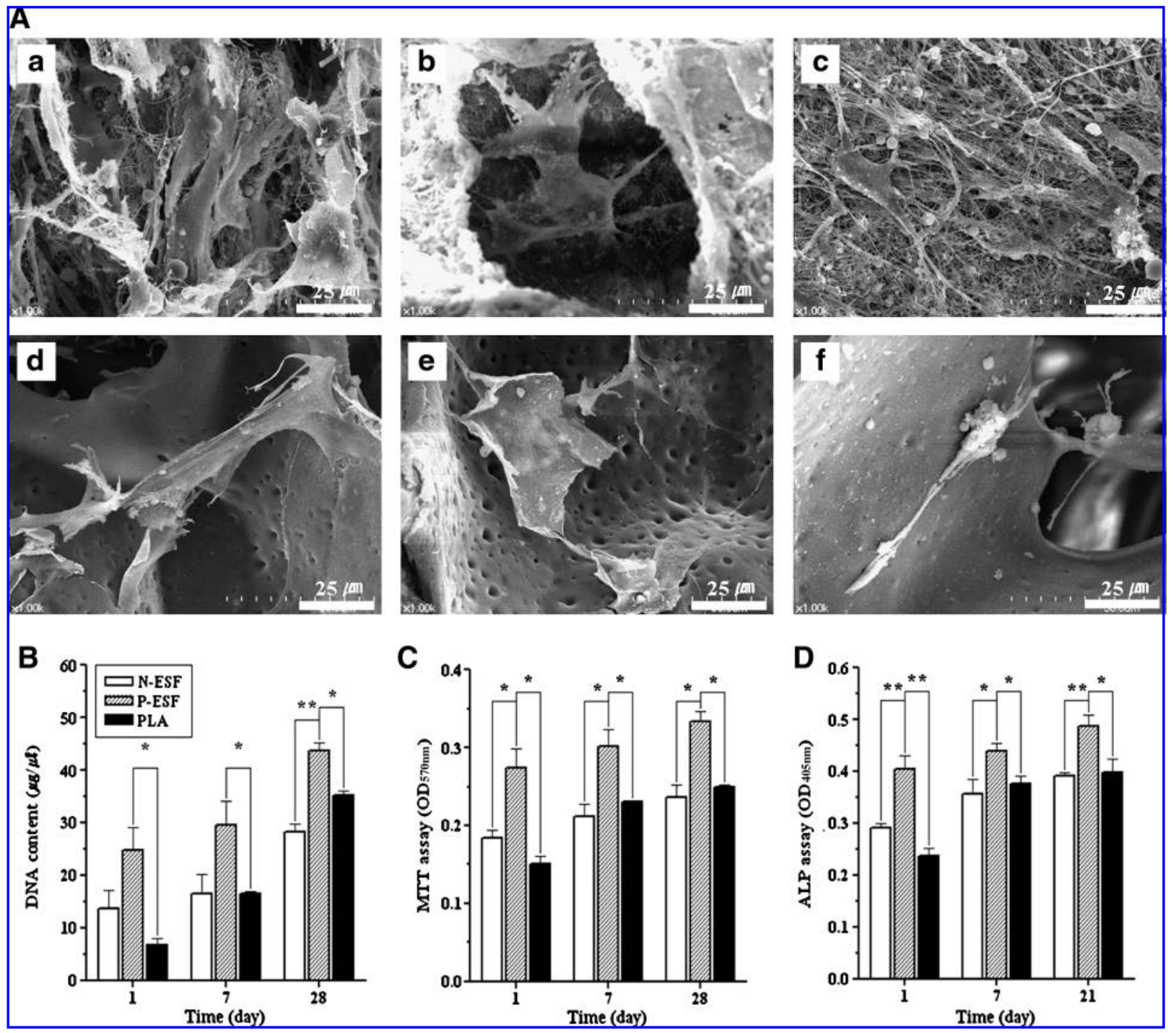

FIG. 2. (A) Scanning electron microscope photographs of osteoblasts on each scaffold: (A-a) 1 day, (A-b) 7 days, and (A-c) 28 days on P-ESF; (A-d) 1 day, (A-e) 7 days, and (A-f) 28 days on PLA scaffold. Quantification of total DNA contents (B) and MTT assay level (C) of cells in the scaffolds were measured at 1, 7, and 28 days after seeding $2.5 \times 10^{5}$ osteoblasts. Alkaline phosphatase activities of cells in the scaffolds were tested at 7, 14, and 21 days after seeding (D). Data are shown as mean \pm standard error of the mean $(n=5)$. ${ }^{*}$ Statistically significant (Student's $t$-test: $\left.{ }^{*} p<0.05,{ }^{* *} p<0.01\right)$. 


\section{Statistical analysis}

The quantitative data were expressed as mean \pm standard error of the mean and analyzed using $T$-test (Student's $t$-test) analysis software (Origin8.0; Origin Lab, Northampton, MA). The differences were considered statistically significant at ${ }^{*} p<0.05$ and ${ }^{* *} p<0.01$.

\section{Results}

\section{Pore size-controllable ESF has favorable} characteristics as a bioactive scaffold

The internal structure of scaffolds was evaluated by SEM (Fig. 1A). N-ESF (Fig. 1A-a), which was fabricated without porogen $\mathrm{NaCl}$ particles, did not show any pores except very tiny spaces around the SF fiber entanglements. However, we could control the pores of ESFs with a salt-leaching method using various sized porogens. The final pore sizes of ESFs were controlled from 0 to $500 \mu \mathrm{m}$ depending on the added porogen sizes (Fig. 1B). The small-sized (S-ESF; Fig. 1A-b), medium-sized (M-ESF; Fig. 1A-c), and large-sized (L-ESF; Fig. 1A-d) ESFs were prepared with 50-100, 100-200, and $300-500 \mu \mathrm{m}$ sized $\mathrm{NaCl}$ during a fabrication process, respectively. Every ESF was composed of SF fiber assemblies of $200-500 \mathrm{~nm}$ in diameter. For biological comparison, a PLA scaffold purchased from BD Falcon (pore size is $150-230 \mu \mathrm{m}$; Fig. 1A-e) was used as a control and compared with M-ESF, which has a similar pore size.

Besides pore size, porosity and water uptake of scaffolds, both of which are important for cytocompatibility, were also measured. Usually, scaffold porosity is defined in the range of $80-90 \%$. When the salt-leaching method is used for obtaining pore structure, the porosity is mainly determined by the amount of porogen. Figure 1C exhibits the porosities of ESFs prepared with $0.15 \mathrm{~g} / \mathrm{mL}$ particles of different sized $\mathrm{NaCl}$. All samples demonstrated $90-93 \%$ porosity regardless of porogen size except N-ESF. The porosity of N-ESF was measured at about $70 \%$, even though the porogen was not added. This was attributed to the tremendous number of tiny spaces created by the fibrous structure, and this porosity value is similar to that of the $2 \mathrm{D}$ fibrous mat fabricated by a common electrospinning process. ${ }^{17}$

Water uptake is closely related to the scaffold porosity because water occupied the vacant space (pore) in the scaffold when the ESF was immersed in the solution. As shown in Figure 1D, the tendency of water uptake was very similar to that of the porosity value. The amount of water adsorbed by ESF with $90 \%$ porosity was approximately $1200 \%$ of its own weight. Therefore, controllability of the pore structure, porosity, and water uptake of new ESF could enable us to tailor scaffolds suitable for specific cell types or tissues needed for regenerative medicine.

\section{Pore size-controllable ESF for bone regeneration in vitro}

The morphologies of cultured cells in the P-ESF $(\mathrm{a}-\mathrm{c})$ and PLA (d-f) scaffolds were evaluated by SEM at 1 day $(a, d)$, 7 days (b, e), and 28 days (c, f) after cell seeding (Fig. 2A). In $\mathrm{N}-\mathrm{ESF}$, no cell was observed inside the scaffold because of the absence of pores. However, many osteoblasts were observed in the middle of both the P-ESF and PLA groups. A greater number of cells were found in P-ESF than in PLA. At
28 days after seeding, osteoblasts inside P-ESF showed five to six extending filopodia, indicating a large attached area to the scaffold compared with cells on PLA.

Cellular proliferation and metabolic activities were measured by total DNA content (Fig. 2B) and MTT assays (Fig. 2C), respectively. Initially, the number of cells on each scaffold steadily increased with time regardless of scaffold type. One day after seeding, PLA showed a significant decrease compared with other groups $(p<0.05)$. One week after seeding, the DNA content and MTT results of PLA surpassed those of N-ESF. Interestingly, P-ESF showed a significant increase of DNA content and MTT level compared with the other scaffold types at any observed time $(p<0.05)$. Differentiation on each scaffold was measured using ALP activities (Fig. 2D). The ALP assay result was similar to previous MTT and total DNA results. Initially, the ALP level of the N-ESF group was higher than that of cells on PLA, although the ALP level of PLA groups was higher than the N-ESF group after 7 days. However, the P-ESF group showed the highest ALP level continuously over the culture period.

Additionally, immunoblotting was performed to evaluate cellular adhesion with antibodies against adhesion-related proteins (Fig. 3). It is obvious that the protein expressions of integrin $\alpha 5, F A K$, its phosphorylated proteins ( $\mathrm{pY}^{397} \mathrm{FAK}$ and $\left.\mathrm{pY}^{925} \mathrm{FAK}\right)$, and phosphorylated $\mathrm{c}-\mathrm{Src}\left(\mathrm{pY}^{416} \mathrm{c}-\mathrm{Src}\right)$ are more intense with P-ESF than other scaffold types at 1 day after seeding.

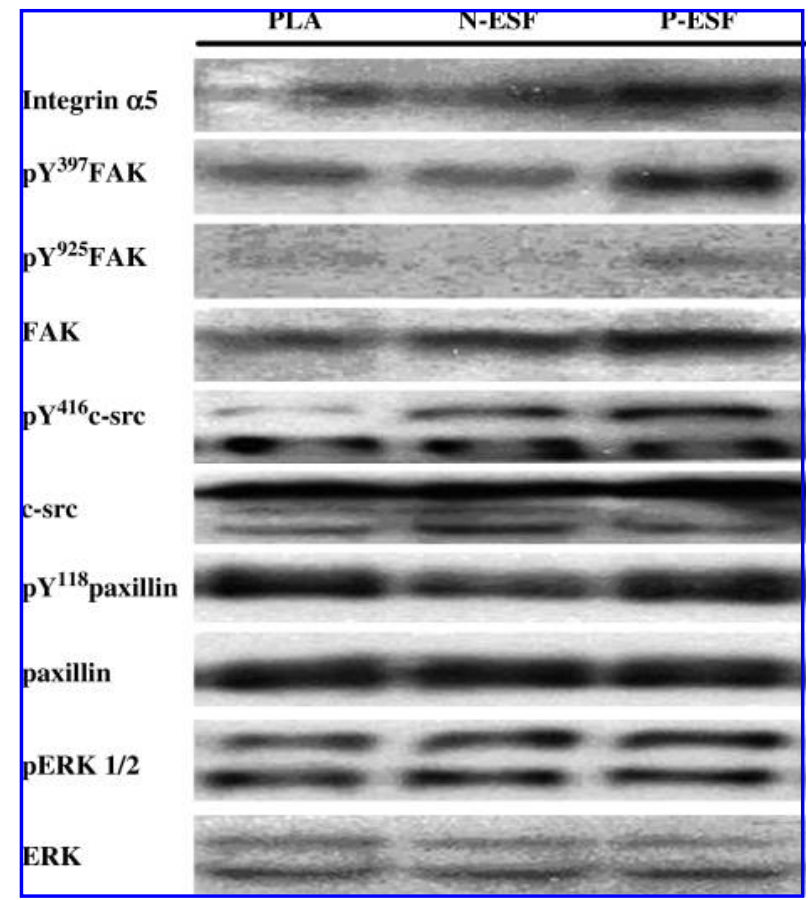

FIG. 3. Western blot analysis with antibodies against adhesion-related proteins, integrin $\alpha 5$, focal adhesion kinase (FAK), c-Src, paxillin, ERK, and phosphorylated forms of FAK, c-Src, paxillin, and ERK, at 1 day after seeding osteoblasts onto PLA, P-ESF, and N-ESF scaffolds. ERK was used as an internal standard. 


\section{Pore size-controllable ESF for bone formation in vivo}

To investigate bone regeneration in vivo, the scaffolds were implanted into punched $(7 \mathrm{~mm}$ diameter) rat calvaria. As negative and positive controls, no defect and only defect (without any scaffolds) were performed, respectively. Samples were obtained at 7 weeks after the operation. Upon macroscopic evaluation (Fig. 4), the defected bone did not reveal any sign of repair; necrosis was observed in the defectonly group. However, in groups treated with PLA and N-ESF scaffolds, the defect zones were regenerated partly with maintaining the original scaffold shapes. But noticeably, the bone defect of one-third of the rats with P-ESF seemed to be mostly recovered with almost absorbed scaffolds at 7 weeks after implantation.

To confirm the regenerated bone area, defected rat calvaria were scanned by $\mu \mathrm{CT}$ (Fig. $4 \mathrm{~A}$ ). The newly generated bone volume was calculated quantitatively using 3D images (Fig. 4B). As expected, the bone volume of the ESF-treated group was good, regardless of the presence of pores, compared with the PLA-treated group. In particular, the P-ESF group yielded higher bone volumes of $78.30 \%$ than the N-ESF $(54.72 \%)$ and PLA (49.31\%) groups, compared with the no defect group (100\%).
The histological evidence of the effect of tissue compatibility and bone regeneration for each scaffold are shown in Figure 5A-E. The vacancies depicted in the figure were caused by xylene treatment during the staining. According to H\&E staining, there was no sign of abnormal tissue response for all scaffold types. Newly regenerated bone cells (deep pink) were observed with inflammation cells in the P-ESF group. Figure 5F denotes the magnified image of cells within the P-ESF group.

\section{Discussion}

The native ECM is a complex fibrous matrix with structural and regulatory proteins. Electrospun porous scaffolds could function like ECM and provide a favorable condition for normal cellular function. In particular, 3D electrospun scaffolds closely mimic native ECM because of the presence of many fine fibers with a small diameter less than several hundred nanometers. ${ }^{13,14}$ Until now, there has been no report of 3D electrospun scaffolds with interconnective macropores.

In this study, macropores among ESF were easily controlled using a salt-leaching method by changing $\mathrm{NaCl}$ particle size. The porosity of the newly developed ESF exceeded
FIG. 4. Bone formation measured with reconstructed threedimensional microcomputed tomography images of rat's calvaria taken at 7 weeks after critical bone defects and implantation of scaffolds (A): (A-a) no defect, (A-b) only defect, (A-c) defect and N-ESF implanted, (A-d) defect and P-ESF implanted, and (A-e) defect and PLA implanted. The dotted circle indicates the original defect. (B) Quantification of the regenerated bone area of the microcomputed tomography images. Data are shown as mean $\pm \mathrm{SD}(n=2$, no defect; $n=5$, only defect and N-ESF; $n=6$, P-ESF and PLA). *Statistically significant (Student's $t$-test: $\left.{ }^{*} p<0.05\right)$.

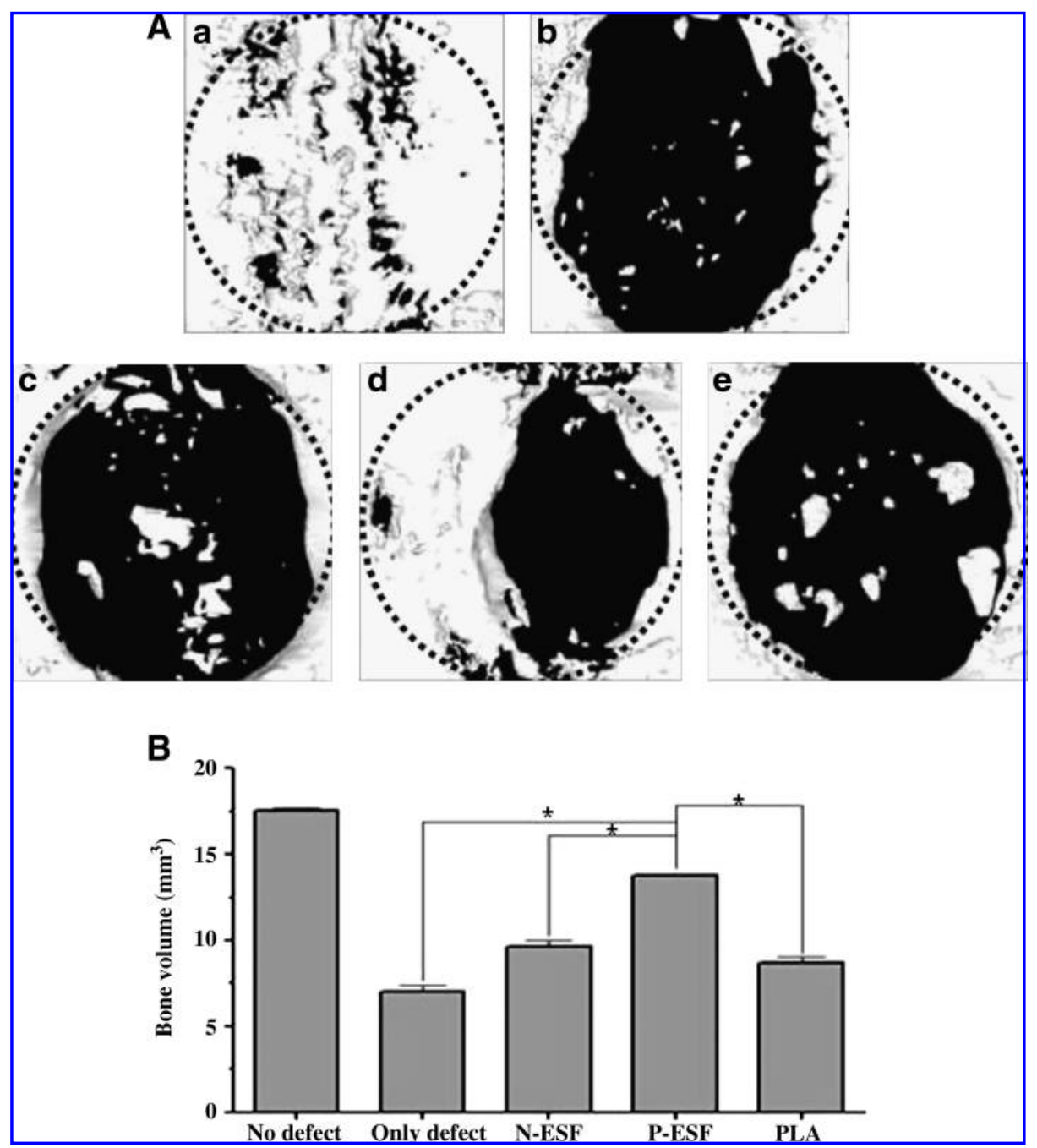




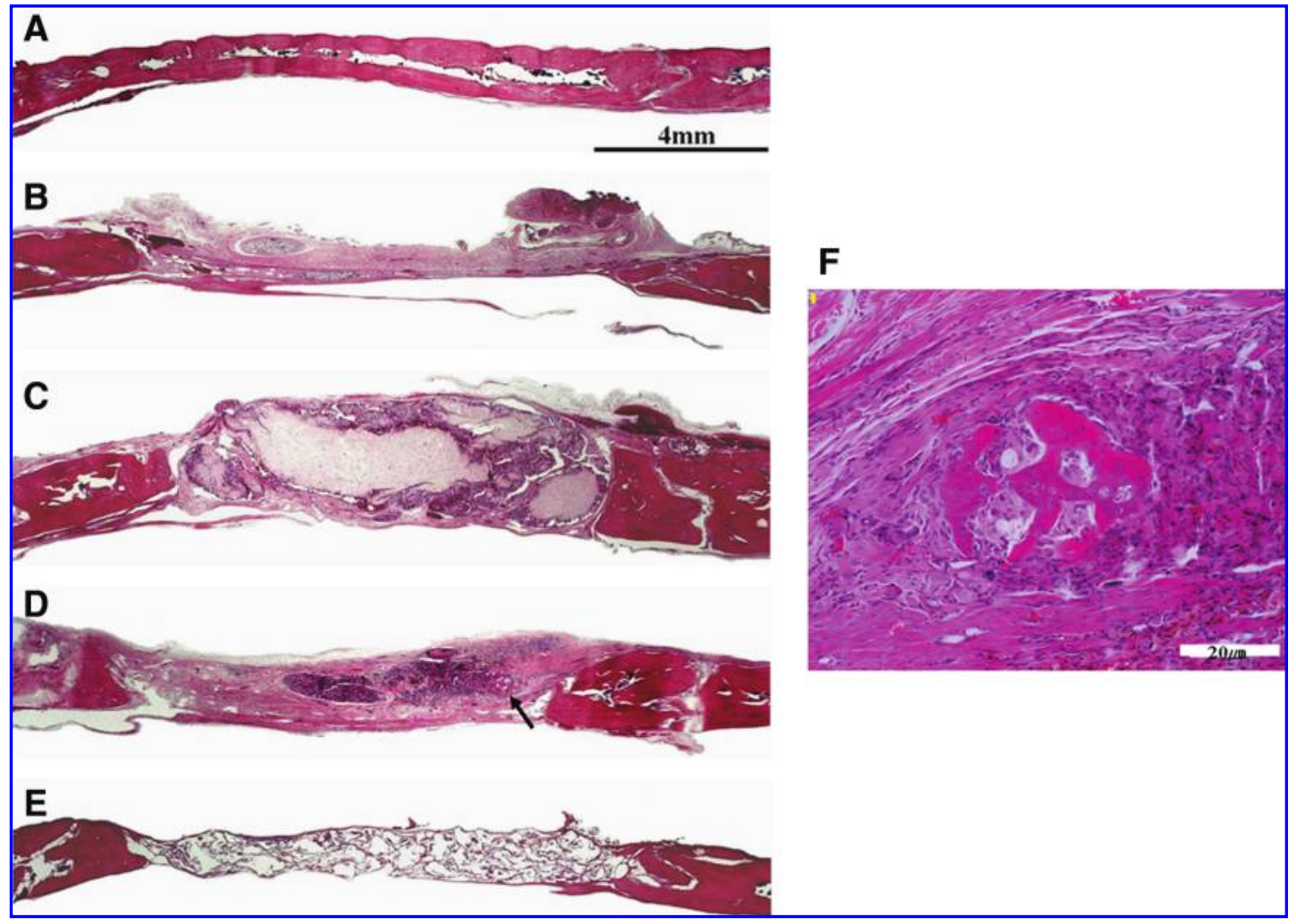

FIG. 5. Histological evaluation of bone formation at 7 weeks after bone defects with hematoxylin and eosin staining. (A) No defect, (B) only defect, (C) defect and N-ESF implanted, (D) defect and P-ESF implanted, and (E) defect and PLA implanted. (F) New bone formation in the P-ESP scaffold. Arrow indicates new bone formation. Color images available online at www.liebertonline.com/ten.

$90 \%$ and it was not significantly changed above $0.10 \mathrm{~g} / \mathrm{mL}$ when the amount of added $\mathrm{NaCl}$ particles exceeded $0.05 \mathrm{~g}$ per unit of ESF dispersion (mL). However, when over $0.20 \mathrm{~g} / \mathrm{mL}$ of $\mathrm{NaCl}$ particles was added, the ESF structure was hardly maintained in the process. Consequently, the optimum amount of $\mathrm{NaCl}$ particles seems to be 0.10 $0.15 \mathrm{~g} / \mathrm{mL}$ (data not shown). For N-ESF, the porosity value of $70 \%$ was attributed to the tremendous number of tiny spaces created by nanofibrous structure, and this porosity value is similar to that of 2D nanofibrous mat fabricated by a common electrospinning process. ${ }^{17}$ Because pore size and porosity of scaffolds have an effect on the mechanical properties, which are important for bone regeneration, ${ }^{21}$ we tested those in dry and water uptake condition (detailed results and discussion are given in Supplemental Material).

We studied ESF with osteoblasts (MC3T3-E1) because silk fibroin is one of several well-known biomaterials for bone regeneration. ${ }^{8,9}$ The osteoblasts were observed in the center of 3D scaffolds such as P-ESF and PLA, even at 4 days after seeding (Fig. 2), which means cells could easily migrate into the scaffolds with suitable pores or porosity. This result was supported by MTT assay and the DNA contents of P-ESF and PLA groups. However, P-ESF showed a higher cellular viability and proliferation compared with PLA, indicating a higher migration of cells along the electrospun fibers in P-ESF. Additionally, the ECM-like electrospun 3D structure of P-ESF may provide a favorable condition for cellular adhesion because many extending filopodia of osteoblasts were observed, which is consistent with a previous report. ${ }^{22}$ Also, we observed greater protein expression of activated adhesion-related proteins, including $\mathrm{FAK}^{23,24}$ in the P-ESF than in PLA using western blot analyses. In this study, P-ESF steadily provided a favorable condition for cell adhesion, migration, and proliferation similar to natural ECM for osteoblasts.

ALP is commonly used as a bone formation marker involved in bone mineral production. ${ }^{25,26}$ The ALP assay result over time was found to be similar to the cell proliferation data. Higher cell density in P-ESF group resulting from the higher cell viability and proliferation during early periods of culture may lead to elevated ALP levels, indicating an enhanced differentiation of osteoblasts. ${ }^{27-29}$

To assess the bone regeneration ability and compatibility in the defect area, we performed critical bone defects in vivo. The scaffolds were implanted into punched rat calvaria and then recovered at 7 weeks after the operation. According to $\mu \mathrm{CT}$ data (Fig. 4), bone is depicted as white and the defect area is black. There is vacant area present in a normal calvarium because the $\mathrm{X}$-ray beam intensity was set high enough to examine the bone regeneration more specifically. In our study, bone regeneration occurred significantly in the N-ESF and P-ESF groups compared with PLA scaffoldtreated groups. In the N-ESF group, the newly formed bone filled the edge of the defect, but the recovered area was insufficient. In case of P-ESF, it was difficult to observe the circular shape of the defect and a significant area was filled 
with new bone. Moreover, bone volume recovery $(78.30 \%)$ in the P-ESF group is comparable to that of N-ESF (54.72\%) and PLA (49.31\%). Regarding N-ESF, the lower bone volume observed compared with P-ESF is due to a lack of porous structure. ESF seems to be a superior biomaterial to PLA with pores because ESF with or without pores showed a higher recovery than PLA with pores. This result suggests that the fibrous structure with macropores is very effective for conducting bone regeneration in vivo. The histological evidence of tissue compatibility and scaffold degradation is shown in Figure 5. In H\&E staining, there was no sign of severe pathologic tissue response for all scaffold types. This may be due to the excellent biocompatibility of ESF and PLA as previously reported. ${ }^{30-33} \mathrm{New}$ bone formation was the most prominent occurrence in the P-ESF group. In Figure 5F, a piece of the regenerated bone was stained pink with the surrounding inflammation cells. Neovascularization, which involves formation of microvascular networks induced by inflammatory response after scaffold implantation, is assumed to be crucial for successful engraftment. ${ }^{34}$ Moreover, the resorption of biomaterial begins in the presence of inflammatory cells. ${ }^{35}$ Our results that the regenerated bone surrounded by inflammatory cells indicate that silk fibroin is a suitable biomaterial from the perspective of biodegradability and tissue healing.

In conclusion, we fabricated an ESF with macropores that can be altered using salt-leaching method. The diameter of electrospun silk fiber was $200-400 \mathrm{~nm}$, providing an ECMlike structure that has high porosity and excellent water uptake. P-ESF showed higher bone formation activities compared with a pore size-matched sponge-type PLA scaffold in vitro and in vivo. A mean of $78.30 \%$ of normal bone area was attained in the P-ESF implantation group at 7 weeks after creating a critical bone defect in rat calvaria. The sponge-type PLA implantation group showed only a $49.31 \%$ bone regrowth area. Therefore, P-ESF may be a good bone substitute for large bone defects because of its ECM-like structure, biocompatibility, and osteoconductivity.

\section{Acknowledgment}

The authors thank the Korea Science and Engineering Foundation (KOSEF) and Rural Development Administration, Republic of Korea, for sponsoring this research through the SRC/ERC Program of MOST/KOSEF (R11-2005-065), and BioGreen21 Program (200810FTH010102001).

\section{Disclosure Statement}

No competing financial interests exist.

\section{References}

1. Betz, V.M., Betz, O.B., Harris, M.B., Vrahas, M.S., and Evans, C.H. Bone tissue engineering and repair by gene therapy. Front Biosci 13, 833, 2008.

2. Christiansen, P. The skeleton in primary hyperparathyroidism: a review focusing on bone remodeling, structure, mass, and fracture. APMIS Suppl 102, 1, 2001.

3. Boivin, G., and Meunier, P.J. The mineralization of bone tissue: a forgotten dimension in osteoporosis research. Osteoporos Int 14 Suppl 3, S19, 2003.
4. Schlichting, K., Dahne, M., and Weiler, A. Biodegradable composite implants. Sports Med Arthrosc 14, 169, 2006.

5. Garofalo, G.S. Autogenous, allogenetic and xenogenetic grafts for maxillary sinus elevation: literature review, current status and prospects. Minerva Stomatol 56, 373, 2007.

6. Chan, B.P., and Leong, K.W. Scaffolding in tissue engineering: general approaches and tissue-specific considerations. Eur Spine J 17 Suppl 4, 467, 2008.

7. Altman, G.H., Diaz, F., Jakuba, C., Calabro, T., Horan, R.L., Chen, J.S., Lu, H., Rchmond, J., and Kaplan, D.L. Silk-based biomaterials. Biomaterials 24, 401, 2003.

8. Minoura, N., Aiba, S.I., Higuchi, M., Gotoh, Y., Tsukada, M., and Imai, Y. Attachment and growth of fibroblast cell on silk fibroin. Biochem Biophys Res Commun 208, 511, 1995.

9. Jin, H.J., Chen, J., Karageorgiou, V., Altman, G.H., and Kaplan, D.L. Human bone marrow stromal cell responses on electrospun silk fibroin mats. Biomaterials 25, 1039, 2004.

10. Mauney, J.R., Nguyen, T., Gillen, K., Kirker-Head, C., Gimble, J.M., and Kaplan, D.L. Engineering adipose-like tissue in vitro and in vivo utilizing human bone marrow and adipose-derived mesenchymal stem cells with silk fibroin 3D scaffolds. Biomaterials 28, 5280, 2007.

11. Min, B.M., Jeong, L., Nam, Y.S., Kim, J.M., Kim, J.Y., and Park, W.H. Formation of silk fibroin matrices with different texture and its cellular response to normal human keratinocytes. Int J Biol Macromol 34, 223, 2004.

12. Badylak, S.F. The extracellular matrix as a scaffold for tissue reconstruction. Semin Cell Dev Biol 13, 377, 2002.

13. Li, W.J., Laurencin, C.T., Caterson, E.J., Tuan, R.S., and Ko, F.K. Electrospun nanofibrous structure: a novel scaffold for tissue engineering. J Biomed Mater Res 60, 613, 2002.

14. Min, B.M., Lee, G., Kim, S.H., Nam, Y.S., Lee, T.S., and Park, W.H. Electrospinning of silk fibroin nanofibers and its effect on the adhesion and spreading of normal by human keratinocytes and fibroblasts in vitro. Biomaterials 25, 1289, 2004.

15. Huang, Z.M., Zhang, Y.Z., Kotaki, M., and Ramakrishna, S. A review on polymer nanofibers by electrospinning and their applications in nanocomposites. Compos Sci Technol 63, 2223, 2003.

16. Karageorgiou, V., and Kaplan, D. Porosity of 3D biomaterial scaffolds and osteogenesis. Biomaterials 26, 5474, 2005.

17. Ki, C.S., Park, S.Y., Kim, H.J., Jung, H.M., Woo, K.M., Lee, J.W., and Park, Y.H. Development of 3-D nanofibrous fibroin scaffold with high porosity by electrospinning: implication for bone regeneration. Biotechnol Lett 80, 405, 2008.

18. Ki, C.S., Kim, J.W., Hyun, J.H., Lee, K.H., Hattori, M., Rah, D.K., and Park, Y.H. Electrospun three-dimensional silk fibroin nanofibrous scaffold. J Appl Polym Sci 106, 3922, 2007.

19. Chen, G., Sato, T., Ushida, T., Ochiai, N., and Tateishi, T. Tissue engineering of cartilage using a hybrid scaffold of synthetic polymer and collagen. Tissue Eng 10, 323, 2004.

20. Bloebaum, R.D., Willie, B.M., Mitchell, B.S., and Hofmann, A.A. Relationship between bone ingrowth, mineral apposition rate, and osteoblast activity. J Biomed Mater Res A 81, 505, 2007.

21. Ikeda, R., Fujioka, H., Nagura, I., Kokubu, T., Toyokawa, N., Inui, A., Makino, T., Kaneko, H., Doita, M., and Kurosaka, $M$. The effect of porosity and mechanical property of a synthetic polymer scaffold on repair of osteochondral defects. Int Orthop 33, 821, 2009.

22. DeMali, K.A., Wennerberg, K., and Burridge, K. Integrin signaling to the actin cytoskeleton. Curr Opin Cell Biol 15, 572, 2003.

23. Azuma, K., Tanaka, M., Uekita, T., Inoue, S., Yokota, J., Ouchi, Y., and Sakai, R. Tyrosine phosphorylation of paxillin 
affects the metastatic potential of human osteosarcoma. Oncogene 24, 4754, 2005.

24. Schlaepfer, D.D., Hanks, S.K., Hunter, T., and van der Geer, P. Integrin-mediated signal transduction linked to Ras pathway by GRB2 binding to focal adhesion kinase. Nature 372, 786, 1994.

25. Gundberg, C.M. Biochemical markers of bone formation. Clin Lab Med 20, 489, 2000.

26. Ogose, A., Hotta, T., Kawashima, H., Hatano, H., Umezu, H., Inoue, Y., and Endo, N. Elevation of serum alkaline phosphatase in clear cell chondrosarcoma of bone. Anticancer Res 21, 649, 2001.

27. Caswell, A.M., Whyte, M.P., and Russell, R.G. Hypophosphatasia and the extracellular metabolism of inorganic pyrophosphate: clinical and laboratory aspects. Crit Rev Clin Lab Sci 28, 175, 1991.

28. Kang, S.K., Kim, K.S., Byun, Y.S., Suh, S.J., Jim, U.H., Kim, K.H., Lee, I.S., and Kim, C.H. Effects of Ulmus davidiana planch on mineralization, bone morphogenetic protein-2, alkaline phosphatase, type I collagen, and collagenase- 1 in bone cells. In Vitro Cell Dev Biol Anim 42, 225, 2006.

29. Declercq, H.A., Verbeeck, R.M., De Ridder, L.I., Schacht, E.H., and Cornelissen, M.J. Calcification as an indicator of osteoinductive capacity of biomaterials in osteoblastic cell cultures. Biomaterials 26, 4964, 2005.

30. Wang, Y., Kim, H.J., Vunjak-Novakovic, G., and Kaplan, D.L. Stem cell-based tissue engineering with silk biomaterials. Biomaterials 27, 6064, 2006.

31. Yang, Y., Chen, X., Ding, F., Zhang, P., Liu, J., and Gu, X. Biocompatibility evaluation of silk fibroin with peripheral nerve tissues and cells in vitro. Biomaterials 28, 1643, 2007.
32. Athanasiou, K.A., Niederauer, G.G., and Agrawal, C.M. Sterilization, toxicity, biocompatibility and clinical applications of polylactic acid/polyglycolic acid copolymers. Biomaterials 17, 93, 1996.

33. Tokiwa, Y., and Calabia, B.P. Biodegradability and biodegradation of poly(lactide). Appl Microbiol Biotechnol 72, 244, 2006.

34. Karayiannakis, A., Zbar, A., Polychronidis, A., and Simopoulos, C. Serum and drainage fluid vascular endothelial growth factor levels in early surgical wounds. Eur Surg Res 35, 492, 2003.

35. Bingle, L., Lewis, C.E., Corke, K.P., Reed, M.W.R., and Brown, N.J. Macrophages promote angiogenesis in human breast tumour spheroids in vivo. Br J Cancer 94, 101, 2005.

Address correspondence to: Hyun Jeong Kim, M.D., Ph.D. Department of Dental Anesthesiology and Dental Research Institute School of Dentistry

Seoul National University 28 Yeongon-dong Jongno-gu

Seoul 110-768

South Korea

E-mail: dentane@snu.ac.kr

Received: May 15, 2009 Accepted: November 10, 2009

Online Publication Date: January 27, 2010 

This article has been cited by:

1. Damien G. Harkin, Karina A. George, Peter W. Madden, Ivan R. Schwab, Dietmar W. Hutmacher, Traian V. Chirila. 2011. Silk fibroin in ocular tissue reconstruction. Biomaterials 32:10, 2445-2458. [CrossRef]

2. Weibo Zhang, Ivy Pruitt Ahluwalia, Robert Literman, David L. Kaplan, Pamela C. Yelick. 2011. Human dental pulp progenitor cell behavior on aqueous and hexafluoroisopropanol based silk scaffolds. Journal of Biomedical Materials Research Part A n/a-n/a. [CrossRef]

3. Pen-Hsiu Grace Chao, Supansa Yodmuang, Xiaoqin Wang, Lin Sun, David L. Kaplan, Gordana Vunjak-Novakovic. 2010. Silk hydrogel for cartilage tissue engineering. Journal of Biomedical Materials Research Part B: Applied Biomaterials 95B:1, 84-90. [CrossRef] 